\title{
Erratum zu: Integration der bodenkundlichen Filter- und Pufferfunktion in die hydrogeologische Vulnerabilitätsbewertung
}

\author{
Tobias Wirsing $\cdot$ Christoph Neukum • Nico Goldscheider $\cdot$ Matthias Maier
}

Online veröffentlicht: 10.09 .2015

(C) Springer-Verlag Berlin Heidelberg 2015

\author{
Erratum zu: Grundwasser - Zeitschrift \\ der Fachsektion Hydrogeologie \\ (2015) 20(2):97-106 \\ DOI 10.1007/s00767-014-0273-5
}

Bedauerlicherweise wurde dieser Beitrag in der letzten Ausgabe nicht in Farbe gedruckt. Wir bitten, dieses Versehen zu entschuldigen.

Die Online-Version des Originalartikels ist unter DOI 10.1007/s00767-014-0273-5 zu finden.

\section{Dipl.-Geoökol. T. Wirsing ( $\bowtie)$}

Institut für Geographie und Geoökologie,

Karlsruher Institut für Technologie (KIT),

Reinhard-Baumeister-Platz 1,

76131 Karlsruhe, Deutschland

E-Mail: tobias.wirsing@kit.edu

Dr. rer. nat. C. Neukum

Lehrstuhl für Ingenieurgeologie und Hydrogeologie,

RWTH Aachen,

Lochnerstr. 4-20,

52064 Aachen, Deutschland

E-Mail: neukum@lih.rwth-aachen.de

Prof. Dr. N. Goldscheider

Institut für Angewandte Geowissenschaften,

Karlsruher Institut für Technologie (KIT),

Kaiserstr. 12,

76131 Karlsruhe, Deutschland

E-Mail: goldscheider@kit.edu

Prof. Dr. M. Maier

Stadtwerke Karlsruhe,

Daxlander Str. 72,

76185 Karlsruhe, Deutschland

E-Mail: matthias.maier@stadtwerke-karlsruhe.de
Zusammenfassung Vulnerabilitätskarten sind Standardwerkzeuge zur Abschätzung der Verschmutzungsempfindlichkeit des Grundwassers. Durch die zunehmende Etablierung in praxisrelevanten Regelwerken wurden sie in den letzten Jahren zum Stand der Technik im Ressourcenmanagement. Dabei wurden von Seiten der Hydrogeologie und der Bodenkunde jeweils eigenständige Verfahren entwickelt, die das Prozessverständnis des eigenen Fachgebiets sehr gut, dasjenige der Nachbardisziplin aber nicht ausreichend berücksichtigen. Da sich von Seiten der Bodenkunde die Datengrundlage zur Bewertung der Schutzfunktion des Bodens in den letzten Jahren hinsichtlich Qualität, Flächenabdeckung und Verfügbarkeit stark verbessert hat, ist es an der Zeit, diesen Datensatz in etablierte hydrogeologische Konzepte zu integrieren. Für Baden-Württemberg wird exemplarisch ein Ansatz vorgestellt, der die bis Ende 2014 flächenhaft im Maßstab 1:50.000 vorliegende Bewertung der Filter- und Pufferfunktion der Böden in die hydrogeologische GLA- und PI-Methode integriert. Durch die neu erstellte Klasseneinteilung in der Gesamtschutzfunktion resultiert eine gleichmäßigere und aussagekräftigere Klassifizierung. Diese führt zu einer differenzierteren Wiedergabe der standörtlichen Verhältnisse und damit zu einer besseren Interpretierbarkeit der Schutzfunktion.

Erratum to: Integration of soil science functions in the hydrogeological assessment of vulnerability

Abstract Vulnerability maps are standard tools for the assessment of groundwater sensitivity to contamination. Due to their increased use in technical guidelines, vulnerability maps have become state-of-the-art tools in resource management. However, most approaches have been developed by hydrogeologists and soil scientists who incorporate the 
understanding of processes from their specific disciplines very well but have limitations in considering processes in other disciplines. A soil-specific database for vulnerability assessment has been significantly improved by soil scientists over the past several years and includes quality, spatial extension and availability. Hence, it is time to integrate this database into hydrogeological concepts. This work presents a vulnerability mapping approach that considers a new soil database that has been available since 2014 for the entire Baden-Württemberg region at a scale of 1:50.000, adapting the well-established GLA and PI methods. Due to the newly-developed classification scheme for the protective function, this approach provides a more balanced and meaningful classification. This leads to a distinct image of the study area and a better interpretation of vulnerability.

Keywords Groundwater vulnerability mapping · PI method - Water resources management · Risk management $\cdot$ Protective function of soils · DVGW W 1001

\section{Einleitung}

Seit Ende der 60er Jahre werden im Rahmen des Ressourcen- und Risikomanagements in der Trinkwassergewinnung immer wieder Verfahren konzipiert und weiterentwickelt, um die Verschmutzungsempfindlichkeit bzw. Vulnerabilität des Grundwassers zu bewerten. Verstärkt eingesetzt werden diese

- seit dem Inkrafttreten der EU-Wasserrahmenrichtlinie (Richtlinie 2000/60/EG des Europäischen Parlaments und des Rats vom 23. Oktober 2000), in der eine Gefährdungsbeurteilung der Grundwasservorkommen gefordert wird,

- seit die World Health Organization mit der Umsetzung des Water-Safety-Plans die Einführung eines prozessorientierten Risikomanagements in der Trinkwasserversorgung fordert (WHO 2006) und

- seit anerkannte Branchenverbände diese Forderungen in landesspezifische Regelwerke aufnehmen (so z. B. der Deutsche Verein des Gas- und Wasserfachs im Rahmen des Technischen Sicherheitsmanagements mit dem Hinweis W 1001 „Sicherheit in der Trinkwasserversorgung - Risikomanagement im Normalbetrieb“; DVGW 2008).

Vulnerabilitätsbewertungen werden aber auch für die landwirtschaftliche Zusatzberatung, die Optimierung von Grundwassermessnetzen und die Gefährdungsabschätzung bei Planung umweltrelevanter Anlagen in der Umweltverträglichkeitsprüfung eingesetzt (Magiera 2000).

Unterschieden wird zwischen der in diesem Beitrag behandelten intrinsischen, also stoffunabhängigen Vulnera- bilität, die eine inhärente Bewertung des dem Grundwasser überlagernden Untergrunds liefert, und der spezifischen Vulnerabilität, die sich auf einen bestimmten Schadstoff bzw. eine bestimmte Schadstoffgruppe bezieht.

\section{Stand der Wissenschaft}

Auf Seiten der Hydrogeologie liegen zahlreiche Bewertungsverfahren vor, die von Magiera (2000) und Zwahlen (2003) zusammengestellt wurden. Am häufigsten wird in Deutschland die GLA-Methode (Hölting et al. 1995) angewandt, die auch bei den geologischen Landesämtern als Standardverfahren eingesetzt wird. Darauf aufbauend wurde die PIMethode von Goldscheider et al. (2000) in Zusammenarbeit mit der Bundesanstalt für Geowissenschaften und Rohstoffe (BGR) und dem Landesamt für Geologie und Rohstoffe Baden-Württemberg (LGRB) entwickelt. Dabei wird die Bewertung des P-Faktors (P: Protective Cover) grundlegend aus der GLA-Methode übernommen. Durch die Einführung des I-Faktors (I: Infiltration conditions) ist die Methode insbesondere auch für den Einsatz in verkarsteten Gebieten geeignet (Goldscheider 2005). Beim Versuch der Validierung unterschiedlicher Vulnerabilitätsbewertungsverfahren mittels numerischer Simulation zeigen die GLA- und die PIMethode gegenüber anderen Verfahren (DRASTIC, EPIK) die besten Übereinstimmungen (Neukum 2006, Neukum et al. 2008). Dies wird darauf zurückführt, dass die Güte der Vulnerabilitätsbewertung maßgeblich von der Berücksichtigung mehrerer hydrogeologisch relevanter Schichten in der ungesättigten Zone abhängt (Neukum 2013).

Auf Seiten der Bodenkunde wurden von den Landesämtern eigenständige Verfahren zur Bewertung der Schutzfunktion des Bodens entwickelt. Seit dem Inkrafttreten des Bundes-Bodenschutzgesetzes (BBodSchG) im Jahr 1999 sind die darin aufgeführten Bodenfunktionen bei umweltrelevanten Planungen zu bewerten. Für Baden-Württemberg liegt bis Ende 2014 für die „Bodenfunktion als Filter und Puffer für Schadstoffe“ (UM BaWü 1995, LUBW 2011) ein flächendeckender Datensatz innerhalb der Bodenkundlichen Karte 1:50.000 (BK50) vor. Die Daten werden den unteren Verwaltungsbehörden in Baden-Württemberg über das WIBAS (Informationssystem Wasser, Immissionsschutz, Boden, Abfall, Arbeitsschutz) im Landesintranet zur Verfügung gestellt.

Bislang wurden keine Versuche unternommen, hydrogeologische und bodenkundliche Bewertungsmethoden in ein gemeinsames Verfahren zu überführen. Während im Jahr 1995, zur Zeit der Publikation der GLA-Methode, in Baden-Württemberg gerade die landesweite Bodenübersichtskarte 1:200.000 (BÜK200) fertig gestellt wurde (Fleck \& Fritz 2010), liegt bis Ende 2014 mit der digitalen, blattschnittfreien BK50 mit zahlreichen Bodenkennwerten 
und funktionen und landesweiter Generallegende fast flächendeckend ein wesentlich umfangreicherer und höher aufgelöster Datensatz vor. Eine Integration dieser Daten in das hydrogeologische Standardverfahren zur Vulnerabilitätsbewertung ist erforderlich. Diesem Nachholbedarf werden sich auch die staatlichen geologischen Dienste im Zuge der „Integrierten geowissenschaftlichen Landesaufnahme“ (GeoLa) in Zukunft stellen müssen (Fleck \& Fritz 2010).

Die Ziele der vorliegenden Arbeit sind daher

- die Stärken und Schwächen der angewandten hydrogeologischen und bodenkundlichen Methoden darzustellen,

- ein Verfahren vorzustellen, wie am Beispiel BadenWürttembergs vorliegende bodenkundliche Methoden und Datensätze bei reduziertem Arbeitsaufwand in den Ansatz der PI-Methode integriert werden können,

- GLA- und PI-Methode hinsichtlich der aufgeführten Bodenarten auf den Stand der aktuellen bodenkundlichen Kartieranleitung (KA5; Ad-hoc-AG Boden 2005) zu bringen,

- auf dieser Grundlage eine verbesserte Klassifizierung für die Gesamtbewertung vorzuschlagen und

- den neuen integrativen Bewertungsansatz mit veränderter Gesamtbewertung an einem Fallbeispiel vorzustellen.

\section{Stärken und Schwächen der existierenden Bewertungsmethoden}

Die Vorteile des bodenkundlichen Verfahrens der LUBW (2011) liegen in der Einbeziehung mehrerer relevanter bodenkundlicher Kennwerte (s. Tab. 1). Diese können auch im Rahmen eigener bodenkundlicher Kartierungen im
Feld angesprochen werden. Im Maßstab 1:50.000 liegt der Datensatz der Filter- und Pufferfunktion in Kürze flächendeckend vor. Der Bodenaufbau wird differenziert bewertet, jedoch nur bis zu einer Tiefe von einem Meter, was ein entscheidendes Defizit in der Bewertung darstellt. Ein weiterer Mangel ist die fehlende Einbeziehung der Grundwasserneubildung, welche gemeinsam mit der nutzbaren Feldkapazität (nFK) die Verweilzeit des Sickerwassers und damit die Abbau- und Adsorptionsprozesse im Boden maßgeblich beeinflusst.

Die Vorteile der GLA- und PI-Methode bei der Bewertung der Grundwasserschutzfunktion liegen in der Berücksichtigung der gesamten ungesättigten Zone, der Grundwasserneubildung als zentrale Eingangsgröße und der Infiltrationsbedingungen (nur PI-Methode), die in Karstsystemen die Verletzlichkeit stark erhöhen können, z. B. durch Oberflächenabfluss im Einzugsgebiet einer Bachschwinde. Wichtige bodenkundliche Parameter werden mit der nutzbaren Feldkapazität des effektiven Wurzelraumes (nFKWe) sowie dem Humusgehalt zwar berücksichtigt, die Güte der über die BK50 digital vorliegenden Informationen wird dabei aber nicht erreicht.

Mit der in Tabelle 1 dargestellten Integration der bodenkundlichen Bewertung des obersten Meters der ungesättigten Zone in die hydrogeologische GLA- und PI-Methode lassen sich die Vorteile der jeweiligen Methoden vereinen. Die flächenhaft vorliegenden Bodeninformationen werden dabei berücksichtigt. Die detaillierte Vorgehensweise bei der Bewertung der bodenkundlichen Filter- und Pufferfunktion sowie bei der hydrogeologischen Vulnerabilitätsbewertung ist den nachfolgenden Ausführungen zum integrativen Bewertungsansatz zu entnehmen.

Tab. 1 Berücksichtigte Kenngrößen in bodenkundlichen und hydrogeologischen Methoden der Vulnerabilitätsbewertung (grün: gute Berücksichtigung der Kenngröße; rot: zu verbessernde Berücksichtigung der Kenngröße)

\begin{tabular}{|c|c|c|c|c|}
\hline Feld $\longrightarrow$ Methode & $\begin{array}{c}\text { Bodenkunde } \\
\text { Filter- \& Pufferfunktion }\end{array}$ & $\begin{array}{l}\text { Hydrogeologie } \\
\text { GLA-Methode }\end{array}$ & $\begin{array}{l}\text { Hydrogeologie } \\
\text { PI-Methode }\end{array}$ & $\begin{array}{c}\text { Integrativer Ansatz } \\
\text { Kombination }\end{array}$ \\
\hline Bodenparameter & $\begin{array}{l}\text { Humusgehalt, Tongehalt, } \mathrm{pH}- \\
\text { Wert, Lagerungsdichte, } \\
\text { Skelettgehalt, Hydromorphie }\end{array}$ & $\begin{array}{l}\text { nFKWe (aus Bodenart), } \\
\text { Humusgehalt (begrenzt) }\end{array}$ & $\begin{array}{c}\mathrm{nFKWe} \\
\text { (aus Bodenart), Humusgehalt } \\
\text { (begrenzt) }\end{array}$ & $\begin{array}{l}\text { Humusgehalt, Tongehalt, pH- } \\
\text { Wert, Lagerungsdichte, } \\
\text { Skelettgehalt, Hydromorphie }\end{array}$ \\
\hline $\begin{array}{c}\text { Differenzierung } \\
\text { der ungesättigten Zone }\end{array}$ & Bodenaufbau bis $1 \mathrm{~m}$ & $\begin{array}{c}\text { Topsoil: } \\
\text { Durchwurzelungszone } \\
\text { Subsoil: Unterlagernde } \\
\text { ungesättigte Zone }\end{array}$ & $\begin{array}{c}\text { Topsoil: } \\
\text { Durchwurzelungszone } \\
\text { Subsoil: Unterlagernde } \\
\text { ungesättigte Zone }\end{array}$ & $\begin{array}{c}\text { Topsoil: Bodenaufbau bis } 1 \mathrm{~m} \\
\text { Subsoil: Unterlagernde } \\
\text { ungesättigte Zone }\end{array}$ \\
\hline Grundwasserneubildung & nicht berücksichtigt & zentrale Eingangsgröße & Zentrale Eingangsgröße & zentrale Eingangsgröße \\
\hline Infiltrationsbedingungen & nicht berücksichtigt & nicht berücksichtigt & $\begin{array}{c}\text { können für } \\
\text { Karstlandschaften } \\
\text { berücksichtigt werden }\end{array}$ & $\begin{array}{l}\text { können für Karstlandschaften } \\
\text { berücksichtigt werden }\end{array}$ \\
\hline
\end{tabular}


Tab. 2 Bewertungsklassen als Filter und Puffer auf Basis der Bodenkartierung von 0 (versiegelt; keine Leistungsfähigkeit) bis 4 (hohe Leistungsfähigkeit) (LUBW 2011)

\begin{tabular}{llllll}
\hline Humusmenge & Tonmenge & \multicolumn{3}{l}{$\mathrm{pH}-\mathrm{max}$} \\
\cline { 5 - 6 }$\left[\mathrm{kg} / \mathrm{m}^{2}\right]$ & {$\left[\mathrm{kg} / \mathrm{m}^{2}\right]$} & $<4,2$ & 4,2 bis $<5$ & 5 bis $<7$ & $\geq 7$ \\
\hline versiegelte Flächen & 0 & & & \\
$<13$ & $<100$ & 1 & 1 & 1 & $1-2$ \\
& $100-300$ & 1 & 1 & $1-2$ & $2-3$ \\
& $>300$ & 1 & 2 & 2 & 3 \\
$13-25$ & $<100$ & 1 & 1 & 1 & 2 \\
& $100-300$ & 1 & 1 & 2 & 3 \\
& $>300$ & 1 & 2 & 3 & 4 \\
$>25$ & $<100$ & 1 & 1 & $1-2$ & $2-3$ \\
& $100-300$ & 1 & $1-2$ & 2 & 3 \\
& $>300$ & 1 & 2 & 3 & 4 \\
\hline
\end{tabular}

Tab. 3 Bewertung der Schutzfunktion des Topsoils mittels nutzbarer Feldkapazität des effektiven Wurzelraums (nFKWe) in GLA- und PIMethode und mittels neu einbezogener Filter- und Pufferfunktion im integrativen Ansatz

\begin{tabular}{lllll}
\hline \multicolumn{2}{l}{ GLA- und PI-Methode } & & Integrativer Ansatz \\
\cline { 1 - 2 } \cline { 5 - 5 } nFKWe $[\mathrm{mm}]$ & Punkte & & FiPu-Funktion & Punkte \\
\hline & & & 4-sehr hoch & 750 \\
$>250$ & 750 & & 3,5-hoch bis sehr hoch) & 550 \\
$>200$ bis 250 & 500 & & 3-hoch & 350 \\
$>140$ bis 200 & 250 & & 2,5-mittel bis hoch & 200 \\
$>90$ bis 140 & 125 & & 2-mittel & 100 \\
$>50$ bis 90 & 50 & & 1,5-gering bis mittel & 50 \\
0 bis 50 (GLA) & 10 & & 1-gering & 25 \\
0 bis 50 (PI) & 0 & & 0-nicht vorhanden & 0 \\
\hline
\end{tabular}

\section{Integrativer Bewertungsansatz}

Der Boden (Topsoil) ist für den Schadstoffrückhalt und -abbau von besonderer Bedeutung. Durch mikro- und makrobiologische Aktivitäten sowie durch die funktionalen Oberflächen und Austauscherplätze an sekundär gebildeten Tonmineralen, angereicherter Organik und pedogenen Oxiden findet im Boden der Hauptanteil der Abbau- und Adsorptionsprozesse statt (Appelo \& Postma 2005, Blume et al. 2010). Besonders leistungsfähige Filter und Puffer sind daher Böden mit hohen $\mathrm{pH}-$ Werten und hohen Humus- und Tongehalten (LUBW 2011). Auf diesen Kennwerten basiert die Bewertungsmatrix in Tabelle 2, aus der die Leistungsfähigkeit der Böden als Filter und Puffer abgeleitet werden kann.

Vor diesem Hintergrund wird in Tabelle 3 die in der Vergangenheit verwendete Kenngröße nFKWe (GLA- und PI-Methode), die stellvertretend für den P-Faktor steht, durch die fundierter berechnete Filter- und Pufferfunktionsbewertung ersetzt, wobei die 750 Punkte auf acht Klassen aufgeteilt werden. Damit wird das Punkteverfahren im integrativen Ansatz grundsätzlich beibehalten.

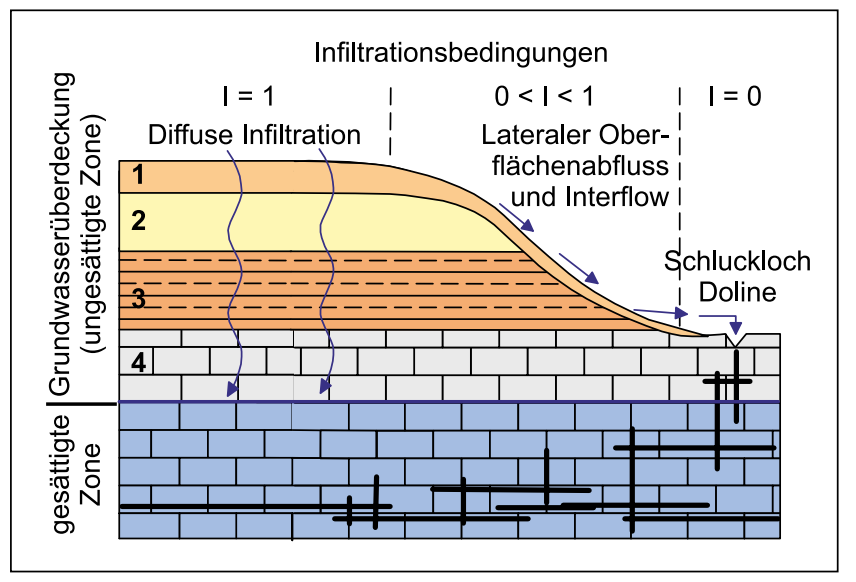

Abb. 1 Der P-Faktor bewertet die Schutzfunktion der Grundwasserüberdeckung, gebildet aus bis zu vier Schichten der ungesättigten Zone; 1: Wurzelraum, 2: tiefere Bodenzone, 3: nicht verkarsteter Untergrund, 4: verkarsteter Untergrund. Der I-Faktor beschreibt den Grad des Umfließens der Grundwasserüberdeckung (Goldscheider 2005)

In Punkte- und Matrixverfahren werden empirisch Parameter ausgewählt, von denen ein maßgeblicher Einfluss auf die Schadstoffverlagerung angenommen wird. Diese werden klassifiziert und über Bewertungssysteme miteinander verrechnet. Für jede Bodeneinheitsfläche ergibt sich eine Punktzahl, die einer Schutzfunktionsklasse zugeordnet werden kann. Neben einem sinnvollen Bewertungssystem stellt dabei die Klassifizierung der Schutzfunktion die größte Herausforderung dar. Diese kann ausschließlich empirisch erfolgen (Magiera 2000).

Der Aufbau der in Abbildung 1 dargestellten Grundwasserüberdeckung (P: Protective cover) aus bis zu vier Schichten Wurzelraum (=Topsoil, 1), tiefere Bodenzone (=Subsoil, 2), nicht verkarsteter Untergrund (3) und verkarsteter Untergrund (4) wird beibehalten, wobei der Topsoil über die Filter- und Pufferfunktion bewertet wird.

Jeder der vier Schichten der ungesättigten Zone wird in Abhängigkeit der Mächtigkeit (M) der in Tabelle 4 aufgeschlüsselte Punktewert zugewiesen, wobei auch komplex aufgebaute Schichten bewertet werden können. Bezüglich des Subsoils werden die in GLA- und PI-Methode verwendeten Bodenarten in die aktuelle Nomenklatur der KA5 (Ad-hoc-AG Boden 2005) übersetzt. Die Bewertung der tieferen ungesättigten Zone (Subsoil), des Gesteins (Lithology) sowie der Klüftigkeit bzw. Verkarstung (Fracturing) wird unverändert übernommen. Die Ermittlung des P-Faktors erfolgt entsprechend der Vorgaben in Tabelle 4. Die aufgeführten und mit Punkten bewerteten Kenngrößen werden über die „Schutzfunktion der Grundwasserüberdeckung" (Gl. 1) miteinander verrechnet. Hinzu kommt ein Faktor, der sich aus der Höhe der Grundwasserneubildung (R: Recharche) ergibt. Im Falle von Artesern erfolgt ein Punktezuschlag. 
Tab. 4 Bestimmung des P-Faktors im Integrativen Ansatz. Integration der Filter- \& Pufferfunktion (LUBW 2011) in die GLA- und PI-Methode (Hölting et al. 1995, Goldscheider et al. 2000), aktualisiert auf die Systematik der KA5 und mit neuer Klassifizierung für die Bewertung der Schutzfunktion. (G stellvertretend für G, Gr, O, X mit Angabe der Bodenartenhauptgruppe)

\begin{tabular}{|c|c|c|c|c|}
\hline \multicolumn{2}{|l|}{ T-Topsoil (effektiver Wurzelraum) } & \multicolumn{3}{|l|}{$\mathrm{R}$ - Recharche (GWNB) } \\
\hline Filter- und Pufferfunktion & $\mathrm{T}$ & Recharche [mm/a] & & $\mathrm{R}$ \\
\hline 4-sehr hoch & 750 & 0 bis 100 & & 1.75 \\
\hline 3,5-hoch bis sehr hoch & 550 & $>100$ bis 200 & & 1.5 \\
\hline 3-hoch & 350 & $>200$ bis 300 & & 1.25 \\
\hline 2,5-mittel bis hoch & 200 & $>300$ bis 400 & & 1 \\
\hline 2-mittel & 100 & $>400$ & & 0,75 \\
\hline 1,5-gering bis mittel & 50 & & & \\
\hline 1-gering & 25 & A-Artesian pressure & & A \\
\hline 0 -kein & 0 & (Artesische Verhältnisse) & & 1.500 \\
\hline \multicolumn{5}{|l|}{$\mathrm{S}$ - Subsoil (ungesättigte Zone) } \\
\hline Bodenart nach KA5 & $\mathrm{S}$ & \multicolumn{2}{|c|}{ Bodenart nach KA5/ Grobbodenanteil } & $\mathrm{S}$ \\
\hline $\mathrm{Tt}$ & 500 & \multicolumn{2}{|c|}{$\mathrm{Su} 3, \mathrm{St} 2, \mathrm{G} 5 \mathrm{t}$} & 75 \\
\hline $\mathrm{Tu} 2, \mathrm{Tl}$ & 400 & \multicolumn{2}{|l|}{$\mathrm{S} 12, \mathrm{G} 5 \mathrm{u}, \mathrm{G} 5 \mathrm{l}$} & 60 \\
\hline Ts2 & 350 & \multicolumn{2}{|l|}{$\mathrm{Su} 2, \mathrm{Su} 2 \mathrm{G} 3-4$} & 50 \\
\hline Tu3 & 320 & \multicolumn{2}{|l|}{ Ss (fS, mS, gS) } & 25 \\
\hline Lt3 & 300 & \multicolumn{2}{|l|}{ Ss G3-4, G5 s } & 10 \\
\hline Tu4, Ts3 & 270 & \multicolumn{2}{|l|}{ G6, Gr6, O6, X6 } & 5 \\
\hline Lt2, Lts, Ut4 & 240 & \multicolumn{2}{|l|}{$\mathrm{H}-$ Torf } & 400 \\
\hline $\mathrm{Lu}$ & 220 & \multicolumn{2}{|l|}{ F - Mudde } & 300 \\
\hline Ls2, Ut3, Ts4 & 200 & \multicolumn{3}{|c|}{ OS - bei erhöhtem Gehalt an org. Substanz } \\
\hline Ls3, Ut2 & 180 & \multicolumn{2}{|c|}{ (n. bei Torf \& Mudde) Zuschlag von: } & OS \\
\hline Uls, Uu, Ls4 & 160 & \multicolumn{2}{|c|}{ h6 } & 100 \\
\hline Slu, St3 & 140 & \multicolumn{2}{|l|}{ h5 } & 75 \\
\hline Us, S14 & 120 & \multicolumn{2}{|l|}{ h4 } & 50 \\
\hline $\mathrm{Su} 4, \mathrm{~S} 13$ & 90 & \multicolumn{2}{|l|}{ h3 } & 25 \\
\hline \multicolumn{2}{|l|}{ L- Lithology (Gestein) } & \multicolumn{3}{|l|}{ F - Fracturing (Klüftigkeit) } \\
\hline Gestein & $\mathrm{L}$ & Struktur & & $\mathrm{F}$ \\
\hline Tonstein, Tonschiefer, Mergelstein, Schluffstein & 20 & ungeklüftet & & 25 \\
\hline \multirow{2}{*}{$\begin{array}{l}\text { Sandstein, Quarzit, vulkanisches Festgestein, Plutonite, } \\
\text { Metamorphite }\end{array}$} & 15 & \multirow{2}{*}{$\begin{array}{l}\text { wenig geklüftet } \\
\text { mittel geklüftet }\end{array}$} & & 4 \\
\hline & & & wenig verkarstet & 1 \\
\hline \multirow[t]{2}{*}{ poröser Sandstein, poröse Vulkanite (z. B. verfestigter Tuff) } & 10 & \multirow{3}{*}{\multicolumn{2}{|c|}{$\begin{array}{ll}\text { stark geklüftet, zerrüttet } & \begin{array}{l}\text { mittel verkarstet } \\
\text { stark verkarstet } \\
\text { nicht bekannt }\end{array}\end{array}$}} & 0,5 \\
\hline & & & & 0,3 \\
\hline Konglomerat, Brekzie, Kalkstein, Kalktuff, Dolomit, Gips & 5 & & & 1 \\
\hline
\end{tabular}

$M$ Mächtigkeit der einzelnen Boden- und Sedimentschichten [m], $B$ Bedrock (Gesteinsuntergrund): $\mathrm{B}=\mathrm{L} \cdot \mathrm{F}$

$P_{T S}$ Schutzfunktion der Grundwasserüberdeckung (Gl. 1)

$$
P_{T S}=\left[T+\left(\sum_{i=1}^{m} \mathrm{~S}_{\mathrm{i}} \cdot \mathrm{M}_{\mathrm{i}}+\sum_{\mathrm{j}=1}^{\mathrm{n}} \mathrm{B}_{\mathrm{j}} \cdot \mathrm{M}_{\mathrm{j}}\right)\right] \cdot R+A
$$

\section{Gesamtbewertung}

Die Gesamtbewertung beschreibt die Ableitung der Gesamtschutzfunktion aus der berechneten Punktezahl $\left(\mathrm{P}_{\mathrm{TS}}\right)$ sowie die darauf aufbauende Bewertung der Vulnerabilität des Grundwassers.

Da bei der Klasseneinteilung der Gesamtschutzfunktion die logarithmische Bewertungsspanne der ursprünglichen PI-Methode in der Regel bei Weitem nicht ausgenutzt wird und da bereits oberhalb von 2.000 Punkten durch die Verweilzeit des Sickerwasser von mehreren Jahren eine sehr hohe Schutzwirkung vorhanden ist, werden in Anlehnung an die Korngrößenklassifizierung nach DIN 14688-1 (2013) halbe Stufen des dekadischen Logarithmus verwendet. Die gewählte Einteilung hat den Vorteil, dass die Klassenabstände auf einer 10er-log-Skala gleich sind. Gleichzeitig kann die in der PI-Methode mit 0 bis 10 Punkten sehr eng bemessene Klasse ,sehr geringe Schutzfunktion“ auf 0 bis 63 Punkte vergrößert werden. Neukum, Hötzl (2006) konnten zeigen, dass gerade die Klasseneinteilung eine sehr wichtige Rolle in der Endbewertung spielt. Tabelle 5 zeigt die gewählte Klasseneinteilung mit Größenordnung der Verweilzeit des Sickerwassers in der ungesättigten Zone.

Bei der Berechnung der intrinsischen Vulnerabilität $(\pi)$ wird die Schutzfunktion der Grundwasserüberdeckung um den I-Faktor, der den Grad des Umfließens der Grundwasserüberdeckung beschreibt, vermindert. Die I-Faktoren 
Tab. 5 Vorschlag einer neuen Klasseneinteilung der Gesamtschutzfunktion im Integrativen Ansatz auf einer halbstufigen dekadisch-logarithmischen Skala

\begin{tabular}{|c|c|c|c|}
\hline $\begin{array}{c}\text { Punktezahl } \\
\text { PTS }_{\text {TS }}\end{array}$ & P-Faktor & $\begin{array}{c}\text { Gesamtschutzunktion } \\
\text { Sickerwassers in der ungesättigten Zone }\end{array}$ \\
\hline 0 bis 63 & 1 & sehr gering & wenige Tage bis Wochen \\
\hline$>63$ bis 200 & 2 & gering & wenige Wochen bis Monate \\
\hline$>200$ bis 630 & 3 & mittel & wenige Monate bis etwa ein Jahr \\
\hline 7630 bis 2000 & 4 & hoch & etwa ein Jahr bis wenige Jahre \\
\hline
\end{tabular}

liegen zwischen 1 (Versickerung erfolgt vollständig als Matrixfluss; gut durchlässige Böden bei geringer Hangneigung) und 0 (Niederschlag gelangt z. B. durch eine Doline im Karst vollständig ungefiltert ins Grundwasser). Die Intrinsische Vulnerabilität ( $\pi$ ) ergibt sich aus der Beziehung

[2] $\pi=P \cdot I$

mit

\section{$P$ aus Tabelle 5}

$I$ aus Tabelle 6

Sie umfasst eine Spanne von 0 bis 5 . Hohe Werte sind mit einer hohen Schutzwirkung der Grundwasserüberdeckung (Boden und tiefere ungesättigte Zone) bzw. mit einer geringen Vulnerabilität des Grundwassers gleichzusetzen (Tab. 6). Niedrigere Werte zeigen eine geringe Schutzfunktion bzw. hohe Vulnerabilität.
Eine bislang unberücksichtigte Kenngröße ist die Amplitude der Grundwasserspiegelschwankung, die im Umfeld größerer Fließgewässer (und in Karstaquiferen) beträchtliche Ausmaße annehmen kann. Hierdurch schwankt einerseits die Mächtigkeit der schützenden Grundwasserüberdeckung, andererseits können Schadstoffe mit der wechselweisen Aufsättigung und dem Leerlaufen der ungesättigten Zone beschleunigt ins Grundwasser gelangen.

\section{Fallbeispiel Einzugsgebiet Wasserwerk Mörscher Wald}

Das südlich von Karlsruhe gelegene Wasserschutzgebiet (WSG) Mörscher Wald liegt im Oberrheingraben, einer geologisch jungen aktiven Störungszone, deren Entstehung im Neogen (früher Alttertiär) mit einer Aufdomung des Gebiets mit Zentrum im südlichen Oberrheingraben (Kaiserstuhl) begann. Zeitgleich mit dem Beginn des Einsinkens der

Tab. 6 Kartenlegende zu Vulnerabilität, P- und I-Faktor

\begin{tabular}{|c|c|c|c|c|c|}
\hline \multicolumn{2}{|c|}{$\begin{array}{c}\text { Vulnerabilitätskarte } \\
\text { Vulnerabilität des } \\
\text { Grundwassers }\end{array}$} & \multicolumn{2}{c|}{$\begin{array}{c}\text { P-Karte } \\
\text { Schutzfunktion der } \\
\text { Grundwasserüberdeckung }\end{array}$} & \multicolumn{2}{c|}{$\begin{array}{c}\text { I-Karte } \\
\text { Grad des Umfließens der } \\
\text { Grundwasserüberdeckung }\end{array}$} \\
\hline Beschreibung & $\pi$-Faktor & Beschreibung & P-Faktor & Beschreibung & I-Faktor \\
\hline extrem & 0 bis 1 & sehr gering & 1 & sehr hoch & $0-0,2$ \\
\hline hoch & $>1$ bis 2 & gering & 2 & hoch & 0,4 \\
\hline mittel & $>2$ bis 3 & mittel & 3 & mittel & 0,6 \\
\hline gering & $>3$ bis 4 & hoch & 4 & gering & 0,8 \\
\hline sehr gering & $>4$ bis 5 & sehr hoch & 5 & sehr gering & 1,0 \\
\hline
\end{tabular}


Bruchschollen im Eozän begann auch die Auffüllung des Grabens mit Süß- und Brackwassersedimenten (Schwarz 2005). Der Hauptteil des Schutzgebiets kann der Grabenscholle zugeschrieben werden. Innerhalb dieser erfolgt die Grundwassergewinnung im etwa $50 \mathrm{~m}$ mächtigen, aus Kiesen und Sanden aufgebauten Oberen Grundwasserleiter. Parallel zum im Osten angrenzenden Schwarzwald trennt eine Hauptverwerfungslinie den äußersten Kilometer des Schutzgebiets in die Vorbergzone ab. Im Bereich des WSG sind hier nur noch teilweise Rheinschotter verbreitet, vermehrt steht Pliozän, Miozän und Oligozän (HGK 2007) unter lokal vorhandenen holozänen Löss- und Lösslehmauflagen an. Somit endet dort der Porengrundwasserleiter.

Naturräumlich und bodenkundlich wird der nordwestliche Teil des Schutzgebiets durch die bewaldete Niederterrasse, mit homogenen, sandig-kiesigen, stark versauerten (Bänder-)Braunerden und Flurabständen von 5 bis 8 Metern aufgebaut (Abb. 2, Profile P1 und P2). Nach Südosten schließt die landwirtschaftlich genutzte Kinzig-Murg-Rinne an. Schotterplattenreste werden von Rinnensystemen umgeben, die sehr heterogene Substrate (schluffig, tonig, sandig, anmoorig) aufweisen. Die Flurabstände betragen nur noch 0

Abb. 2 Intrinsische Vulnerabilität im Einzugsgebiet des Wasserwerks Mörscher Wald (integrativer Ansatz)

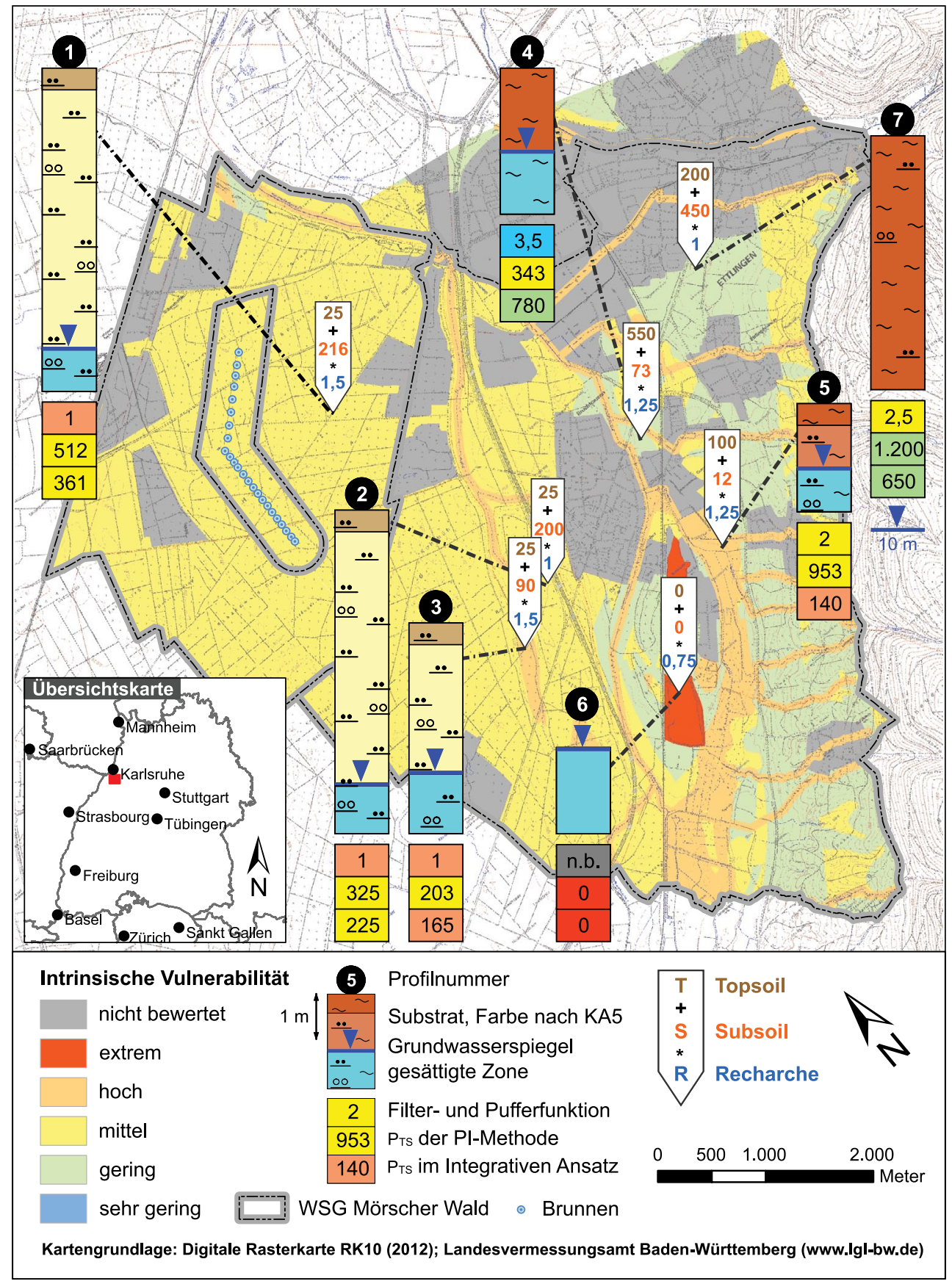




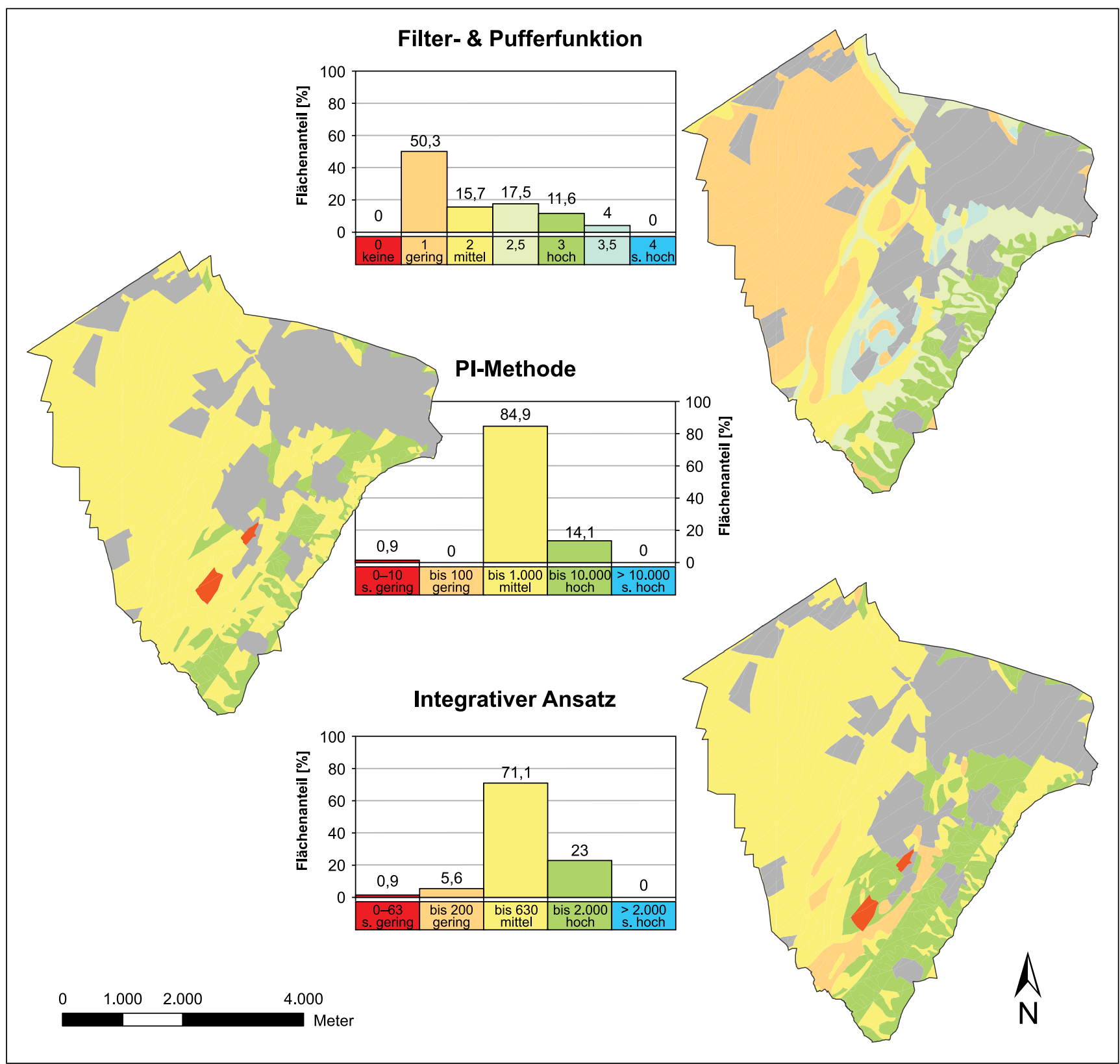

Abb. 3 Schutzfunktionskarten und Häufigkeitsverteilungen für die diskutierten Bewertungsmethoden im Einzugsgebiet des Wasserwerks Mörscher Wald

bis 3 Meter (Abb. 2, P3-P5). Im Bereich der Vorbergzone steigt das Gelände stärker als die Flurabstände an. Die ungesättigte Zone wird von Löss- und Lösslehmdecken sowie lokal von Buntsandsteinhangschutt aufgebaut (Abb. 2, P7).

Abbildung 3 stellt für das Einzugsgebiet des Wasserwerks Mörscher Wald die Filter- und Pufferfunktion des Bodens der Schutzfunktion der Grundwasserüberdeckung von PI-Methode und integrativem Ansatz gegenüber. Da sich die GLA-Methode im betrachteten Porengrundwasserleiter nur hinsichtlich der Klasseneinteilung unterscheidet, wurde auf die Darstellung verzichtet. Die Filter- und Pufferfunktion weist die größte Bandbreite berechneter Klassen auf. Die geringere Bandbreite berechneter Vulnerabilitätsklassen von PI-Methode und integrativem Ansatz ist dem geologisch recht einheitlich aufgebauten Untersuchungsgebiet geschuldet.

Da im Untersuchungsgebiet Flächen mit geringer Funktionserfüllung meist aber über große Flurabstände verfügen, fällt deren Bewertung in PI-Methode und integrativem Ansatz günstiger aus. Böden mit sehr hoher Eignung als Filter und Puffer hingegen liegen in der Kinzig-Murg-Rinne und verfügen meist über sehr geringe Flurabstände, weshalb sie in PI-Methode und integrativem Ansatz abgewertet werden. 
Durch die Einbeziehung der Filter- und Pufferfunktion in den integrativen Ansatz unterscheidet sich dieser in einigen Bereichen von der PI-Methode: Bereiche der Kinzig-MurgRinne sowie der Vorbergzone werden durch die hohe Leistungsfähigkeit der Böden als Filter und Puffer eine Klasse aufgewertet, Bereiche der Kinzig-Murg-Rinne mit sauren $\mathrm{pH}-$ Werten und sandig-kiesigem Substrat bei gleichzeitig geringen Flurabständen werden eine Klasse abgewertet. Während bei der PI-Methode (auch der dekadisch logarithmischen Klasseneinteilung geschuldet) $85 \%$ der Schutzgebietsfläche mit mittlerer Schutzfunktion bewertet werden und die übrige Fläche (mit Ausnahme der Baggerseen) mit hoher Schutzfunktion bewertet wird, differenziert der integrative Ansatz stärker.

Abbildung 2 zeigt die Vulnerabilitätsbewertung nach vorgestellter Methode, wobei exemplarisch für sieben Flächen die Berechnung des P-Faktors aus den Punktewerten von Topsoil (T), Subsoil (S) und Recharge (R) in Pfeilform dargestellt ist. Für die exemplarischen Bodenprofile (P1 bis P7) wurden die Bewertungen nach Filter- und Pufferfunktion, PI-Methode und integrativem Ansatz angegeben. Da der I-Faktor in dem ebenen, unverkarsteten Einzugsgebiet stets 1 beträgt, ist mit dem P-Faktor die Vulnerabilitätsbewertung vollständig abgeschlossen.

Der nordwestliche Teil des Schutzgebiets verfügt wegen seiner bis zwei Meter tief entkalkten, stark versauerten und sandig-kiesigen Substrate (P1 und P2) im obersten Profilmeter nur über eine sehr geringe Leistungsfähigkeit als Filter und Puffer. Wegen der großen Grundwasserflurabstände wird die Schutzfunktion im integrativen Ansatz mit 361 Punkten (P1) niedriger bewertet als in der PI-Methode (mit 512 Punkten).

In der im Südosten anschließenden Kinzig-Murg-Niederung mit geringen Grundwasserflurabständen befinden sich die Profile 3 bis 5. Das der Niederterrasse ähnliche P3, das aber eine geringer mächtige ungesättigte Zone aufweist, wird nach PI-Methode mit 203 Punkten noch immer als mittel eingestuft, mittels Integriertem Ansatz und 165 berechneten Punkten ist die Schutzfunktion nur noch gering. Andere Rinnenabschnitte werden durch die PI-Methode durchweg mit mittlerer Schutzwirkung klassifiziert (P4: 343, P5: 953). Das ton- und humusreichere P4 wird demgegenüber aufgewertet (hohe Schutzwirkung; 780 Punkte) und das sandigere, gering humose P5 abgewertet (geringe Schutzwirkung; 140 Punkte). Bei Fläche P6 handelt es sich um eine Grundwasserblänke - eine Schutzwirkung überlagernder Schichten ist nicht vorhanden.

Das Ausgangssubstrat der Böden der Vorbergzone ist Lösslehm (P7) der zu unterschiedlichen Anteilen mit Hangschutt aus Buntsandstein durchsetzt ist. Die Einbeziehung der mittel bis guten $(2,5)$ Leistungsfähigkeit als Filter und Puffer führt zu einer Abwertung der Schutzfunktion im inte- grativen Ansatz (600 Punkte; hohe Schutzwirkung) gegenüber der PI-Methode (1.200 Punkte, hohe Schutzwirkung). Aus dem Schwarzwald herabziehende Bachbetten werden durch die in diesem Bereich dominierenden influenten Verhältnisse mit hoher Vulnerabilität eingestuft.

\section{Fazit}

Da sich die Datengrundlage zur Bewertung der Schutzfunktion der Grundwasserüberdeckung in den letzten Jahren hinsichtlich Qualität, Flächenabdeckung und Verfügbarkeit stark verbessert hat, ist es notwendig diese Daten in etablierte Verfahren einzubinden. Mit dem vorgestellten integrativen Ansatz gelingt es, bislang eigenständige Bewertungsverfahren der Hydrogeologie und der Bodenkunde zu kombinieren und die Stärken beider Fachdisziplinen zu vereinen. Der bis Ende 2014 in Baden-Württemberg flächendeckend vorliegende Datensatz der Filter- und Pufferfunktion der Böden wird als Modul in die in der Hydrogeologie etablierten GLA- und PI-Methode integriert. Da die in der GLA- und PI-Methode bislang nötige Ermittlung des effektiven Wurzelraums und dessen nutzbarer Feldkapazität entfällt, erhöht der vorgestellte Ansatz nicht nur die Datenqualität, sondern ist auch einfacher durchzuführen. Die vorgeschlagene Klassifizierung der Gesamtschutzfunktion führt zu einer größeren Differenzierung der bewerteten Flächen und damit zu einer besseren Interpretierbarkeit.

\section{Literatur}

Ad-hoc-AG Boden: Bodenkundliche Kartieranleitung (KA5), 5. Aufl., 438 S. Schweizerbart'sche Verlagsbuchhandlung, Hannover (2005)

Appelo, C.A.J., Postma, D.: Geochemistry, groundwater and pollution, 2. Aufl., S 668. A.A. Balkema Publishers, Amsterdam (2005)

Blume, H.-P., Brümmer, G.W., Horn, R., Kandeler, E., Kögel-Knabner, I., Kretzschmar, R., Stahr, K., Wilke, B.-M.: Scheffer/Schachtschabel - Lehrbuch der Bodenkunde, 16. Aufl., 569 S. Springer Verlag, Heidelberg (2010)

DIN 14688-1: Geotechnische Erkundung und Untersuchung - Benennung, Beschreibung und Klassifizierung von Boden, Teil 1: Benennung und Beschreibung. (2013)

DVGW: Technische Mitteilung, Hinweis W 1001 „Sicherheit in der Trinkwasserversorgung - Risikomanagement im Normalbetrieb“, 19 S. Bonn (2008)

Fleck, W., Fritz, C.: Digitale Bodenkarte von Baden-Württemberg 1: 50 000- Neue Wege in der bodenkundlichen Landesaufnahme. LGRB-Informationen 25, 83-94; Freiburg i. Br. (2010)

Goldscheider, N.: Karst groundwater vulnerability mapping - application of a new method in the Swabian Alb. Hydrogeology Journal 13, 555-564 (2005)

Goldscheider, N., Klute, M., Sturm, S., Hötzl, H.: The PI method - a GIS-based approach to mapping groundwater vulnerability with special consideration of karst aquifers. Z. Angew. Geol. 46(3), 157-166 (2000) 
HGK | Umweltministerium Baden-Württemberg \& Ministerium für Umwelt, Forsten und Verbraucherschutz Rheinland-Pfalz: Hydrogeologische Kartierung und Grundwasserbewirtschaftung im Raum Karlsruhe-Speyer (2007)

Hölting, B., Haertle, T., Hohberger, K.-H., Nachtigal, K.H., Villinger, E., Weinzierl, W., Wrobel, J.-P.: Konzept zur Ermittlung der Schutzfunktion der Grundwasserüberdeckung. Geol. Jahrb. C 63, 5-24; Hannover (1995)

LUBW: Bewertung von Böden nach ihrer Leistungsfähigkeit. Leitfaden für Planungen und Gestattungsverfahren. Bodenschutz 23, Karlsruhe (2011)

Magiera, P.: Methoden zur Abschätzung der Verschmutzungsempfindlichkeit des Grundwassers. Grundwasser 5(3), 103-114 (2000)

Neukum, C.: Ermittlung eines Validierungsparameters zum Vergleich von Vulnerabilitätskonzepten in Karstgebieten. 154 S., Diss. Univ. Karlsruhe (2006)

Neukum, C.: Eine Übersicht zu Methoden und Anwendungen der Validierung von Vulnerabilitätsbewertungen. Grundwasser 18(1), 15-24 (2013)
Neukum, C., Hötzl, H.: Standardization of vulnerability maps. Environ. Geol. Vol. 51(5), 689-694 (2006)

Neukum, C., Hötzl, H., Himmelsbach, T.: Validation of vulnerability mapping methods by field investigations and numerical modelling. Hydrogeology Journal 16(2), 641-658 (2008)

Schwarz, M.: Evolution und Struktur des Oberrheingrabens - quantitative Einblicke mit Hilfe dreidimensionaler thermomechanischer Modellrechnungen. Diss. Univ. Freiburg (2005)

UM BaWü - Ministerium für Umwelt Baden-Württemberg: Bewertung von Böden nach ihrer Leistungsfähigkeit - Leitfaden für Planungen und Gestattungsverfahren. 57 S. Luft, Boden, Abfall 31; Stuttgart (1995)

WHO - World Health Organization: Guidelines for drinking-water quality. 595 S., Genf. Resource document (2006)

Zwahlen, F. (Hrsg.): Vulnerability and Risk mapping for the protection of carbonate (karst)aquifers. Final report COST action 620. $315 \mathrm{~S}$., Resource document. European Communities, Luxembourg (2003) 